\title{
Characteristics of Patients With a Significant Stenosis in a Conventional Coronary Angiogram With a Normal Multi-Detector Computed Tomographic Coronary Angiogram
}

\author{
Hae Chang Jeong, ${ }^{1}$ MD, Youngkeun Ahn, ${ }^{1}$ MD, Myung Ho Jeong, ${ }^{1}$ MD, \\ Jong Won Chung, ${ }^{1}$ MD, Jung Sun Cho, ${ }^{1}$ MD, Du Sun Shim, ${ }^{1}$ MD, \\ Nam Sik Yoon, ${ }^{1}$ MD, Jae Yeun Moon, ${ }^{1}$ MD, Kye Hun KIm, ${ }^{1}$ MD, \\ Hyung Wook Park, ${ }^{1}$ MD, Young Joon HonG, ${ }^{1}$ MD, Ju Han KIm, ${ }^{1}$ MD, \\ Jeong Gwan CHO, ${ }^{1} \mathrm{MD}$, and Jong Chun PARK, ${ }^{1} \mathrm{MD}$
}

\section{SUMMARY}

Multi-detector computed tomography (MDCT) has high diagnostic value for detecting or excluding coronary artery stenosis. However, conventional coronary angiograms (CCA) are occasionally required in patients having persistent chest pain with normal MDCT.

We retrospectively analyzed 90 patients who underwent CCA due to persistent chest pain with normal MDCT. The patients were classified into patients having more than 50\% diameter stenosis in CCA (false negative, group I: $n=14,62.6 \pm 7.5$ years, 7 males) and those having less than 50\% diameter stenosis (true negative, group II: $n=76,52.1 \pm 12.0$ years, 42 males). Significant stenosis was observed in 9 patients at the left anterior descending artery, 4 at the right coronary artery, and 1 at the left circumflex artery in group I. Group I patients were older than group II patients $(63 \pm 8$ versus $52 \pm 12$ years, $P<0.001)$. There were more patients with hypertension and smoking in group I (64.3\% versus $7.9 \%$, $35.7 \%$ versus $3.9 \%, P<0.001, P<0.001$, respectively). The levels of uric acid and homocysteine were higher in group I than in group II $(5.7 \pm 1.5$ versus $4.6 \pm 1.2 \mathrm{mg} / \mathrm{dL}, 9.6$ \pm 3.1 versus $7.4 \pm 2.5 \mathrm{~mol} / \mathrm{L}, P=0.008, P=0.010$, respectively). There were more ST or $\mathrm{T}$ changes in the electrocardiograms in group I (35.7\% versus $1.3 \%)(P<0.001)$. In multivariate analysis, a history of hypertension, uric acid levels, and ischemic evidence in the electrocardiogram were independent factors for a false negative of MDCT (odds ratio $11.11,4.76,1.81,95 \%$ confidence interval 4.67 to $10.00,1.41$ to $1.61,1.05$ to $3.33, P=$ $0.009, P=0.012, P=0.046$, respectively).

In certain situations, the findings of coronary stenosis by MDCT do not always correlate with that of CCA. (Int Heart J 2009; 50: 13-22)

Key words: Coronary artery disease, CT angiogram, Conventional coronary angiogram

From ${ }^{1}$ The Heart Center of Chonnam National University Hospital, Cardiovascular Research Institute of Chonnam National University, Gwangju, Republic of Korea.

Address for correspondence: Youngkeun Ahn, MD, The Heart Center of Chonnam National University Hospital, Cardiovascular Research Institute of Chonnam National University, 8 Hak Dong, Dong Ku, Gwangju 501-757, Republic of Korea.

This work was supported by the stem cell research program of the Ministry of Science \& Technology (grant M10641450001-06N4145-00110).

Received for publication July 10, 2008

Revised and accepted September 22, 2008. 
Coronary artery disease represents a leading cause of death and health care expenditure in industrialized countries. ${ }^{1,2)}$ Establishing its anatomic diagnosis requires conventional coronary angiography (CCA), a procedure that is costly and carries risks and discomfort. ${ }^{3,4)}$ Contrast-enhanced multi-detector-row computed tomography (MDCT) is now capable of providing high-quality noninvasive views of cardiac anatomy and 'instant' noninvasive coronary angiography. With current generation 64-slice scanners, MDCT can be performed in most patients with minimal patient discomfort and high diagnostic accuracy. ${ }^{5,6)}$ MDCT may obviate the need for invasive diagnostic CCA in patients with borderline symptoms or equivocal noninvasive testing.

Single-center studies have reported sensitivities between $85 \%$ and $100 \%$, specificities between $50 \%$ and $95 \%$, positive predictive values between $75 \%$ and $93 \%$, and negative predictive values between $75 \%$ and $99 \%$ for the detection of obstructive coronary lesions using 16-row MDCT scanners. ${ }^{7,8)}$ However, MDCT has disadvantages too. Calcified vessels are still difficult to assess, as is the accurate evaluation of implanted coronary stents. The volume of contrast material required for proper opacification limits the use of MDCT in patients with renal dysfunction. ${ }^{9)}$ Finally, CCA is occasionally required in patients having persistent chest pain with normal MDCT findings. The purpose of this study was to elucidate the characteristics of patients with a significant stenosis in CCA using normal MDCT coronary angiography.

\section{Methods}

Study population and study design: Between January 2006 and July 2007, 5,207 patients with atypical chest pain underwent cardiac MDCT at Chonnam National University Hospital. Among these patients, 492 patients with abnormal MDCT findings underwent CCA and percutaneous coronary intervention (PCI). The other 90 patients with a normal MDCT coronary angiogram (defined as a total calcium score of zero and the absence of critical stenosis in both coronary arteries) complained of recurrent chest pain. These patients underwent diagnostic CCA to determine the cause of the chest pain. All patients underwent a CT angiogram and CCA within a relatively short interval (mean, $13 \pm 6$ days). Fourteen patients had significant stenosis and 76 patients had no significant stenosis in the CCA. The CCA was interpreted by a board-certified cardiologist. Diameter stenosis was assessed by quantitative coronary angiography (QCA). Diameter narrowing of more than $50 \%$ was considered to be significant. Lesion type was determined according to the criteria of the American College of Cardiology/American Heart Association (ACC/AHA). Patients with significant stenosis in CCA were assigned to group I (14 patients, $63 \pm 8$ years, 7 males) 
while those with no significant stenosis were assigned to group II (76 patients, $52 \pm 12$ years, 42 males). Age, sex, body mass index, risk factors for coronary artery disease (hypertension, diabetes, smoking, hyperlipidemia, family history of ischemic heart disease), clinical impression of patients, past medication [aspirin, clopidogrel, beta-blockers, angiotensin converting enzyme inhibitors, angiotensin receptor blockers, statins], blood chemistry (lipid profiles, inflammatory markers, cardiac enzymes, N-terminal pro-B-type natriuretic peptide (NTproBNP)), electrocardiographic (ECG), echocardiographic, and CCA findings were analyzed.

Cardiac MDCT angiography: In the absence of contraindications [contraindication to intravenous contrast agents (contrast allergy) or elevated serum creatinine ( $>1.3 \mathrm{mg} / \mathrm{dL})$ or atrial fibrillation or frequent ventricular ectopy (>10 extra systoles per minute), or a heart rate $>90 \mathrm{bpm}$ ], all patients with a resting heart rate of $>70 \mathrm{bpm}$ received 50-100 $\mathrm{mg}$ of metoprolol orally 1-2 hours before the
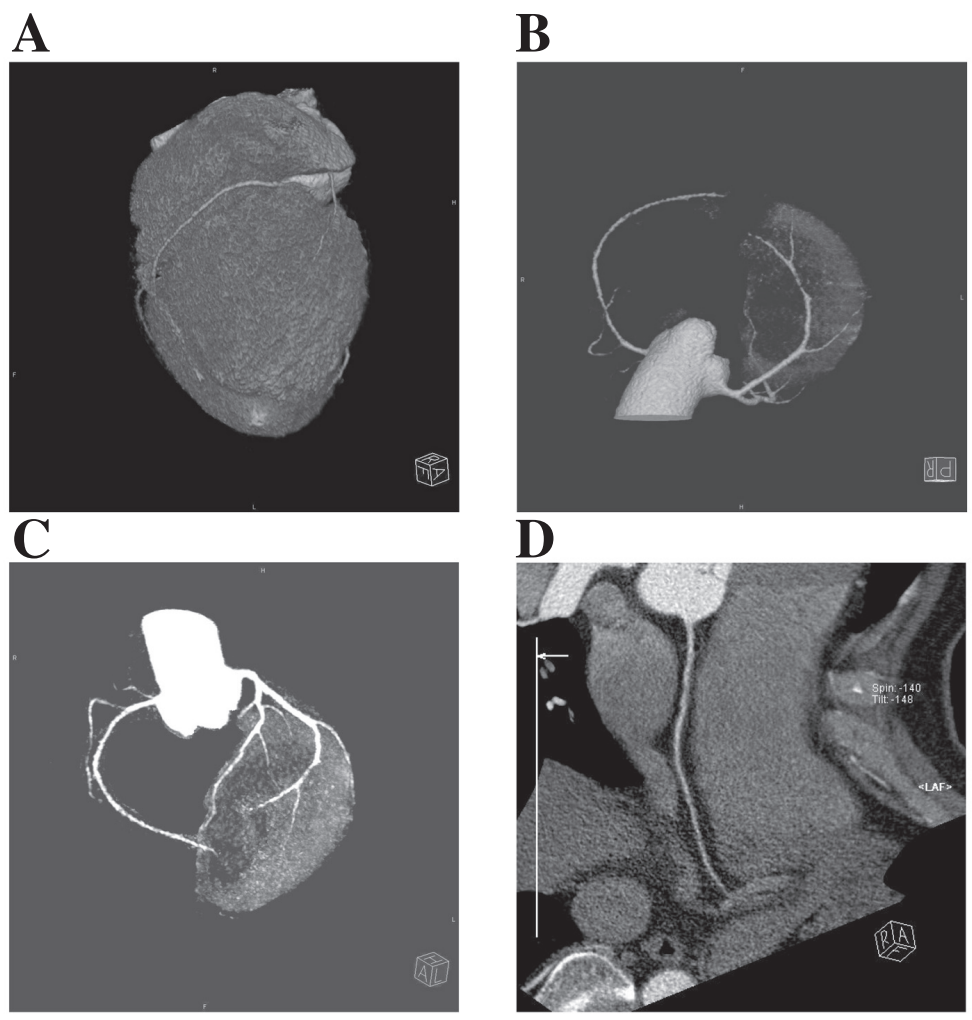

Figure 1. Example of mismatch of stenosis between multi-detector computed tomogram (MDCT) and conventional coronary angiogram (CCA) in a 58 year-old male patient. There was no significant stenosis of either coronary artery on 3-dimensional reconstruction by MDCT $(\mathbf{A}, \mathbf{B}, \mathbf{C})$. Close-up multiplanar reformation image of the right coronary artery (RCA) revealed no significant stenosis (D). 
scan. Blood pressure and pulse were rechecked immediately prior to performing the procedure. CT examinations were performed with a two-phase, contrast-enhanced, ECG-gated, MDCT scanner (Sensation Cardiac 64, Siemens, Forchhe$\mathrm{im}$, Germany) set at a $0.75-\mathrm{mm}$ section thickness with a gantry rotation time of $330 \mathrm{msec}$ and a kernel value of B25f. The tube current was $800 \mathrm{mAs}$ at $120 \mathrm{kVP}$. The pitch, defined as the ratio of the table feed in one rotation over the detector coverage in the transverse direction, was determined as 0.2 . The scan delay was determined using the bolus tracking technique: when a threshold of 120 Hounsfield Units (HU) was reached in the ascending aorta at the level of the origin of the coronary arteries, a delay of 5 seconds was applied before the scan was initiated. Serial CT scanning in the axial plane, together with an ECG triggered examination, was performed from the level of the left ventricular apex after a
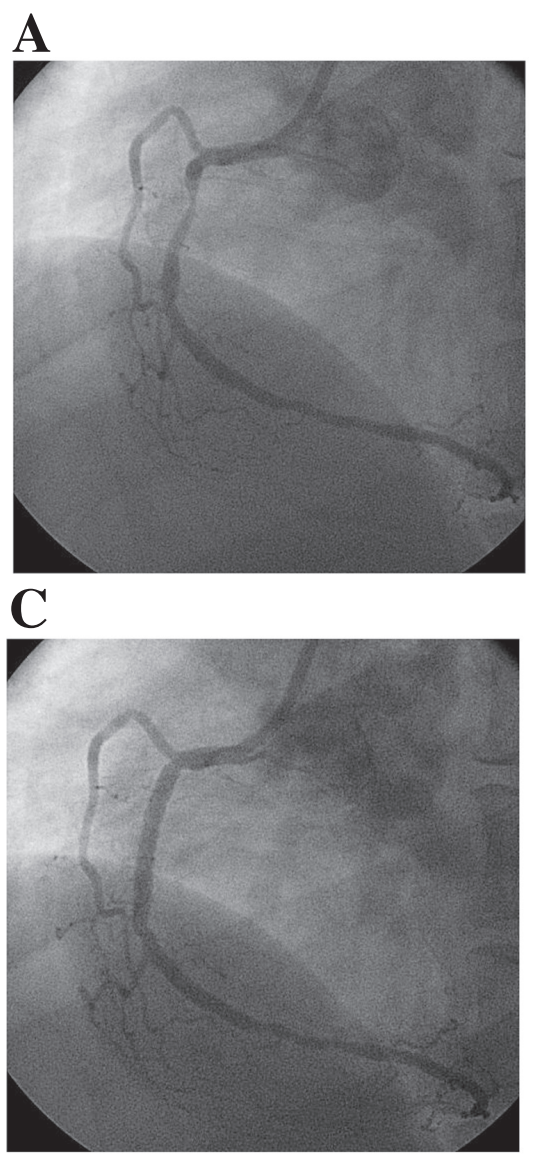

Figure 2. Significant stenosis of the mid-RCA was seen on CCA (A). Intravascular ultrasonogram (IVUS) revealed significant mixed plaque in the mid-RCA $(\mathbf{B})$. IVUS-guided stenting $(3.0 \times$ $23 \mathrm{~mm}$ Cypher) was performed for this lesion. The final coronary angiogram showed good distal flow without residual stenosis (C). IVUS findings after stent implantation (D).

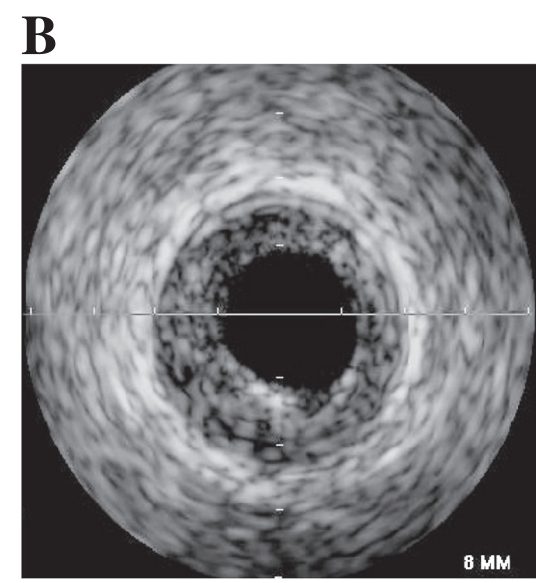

D

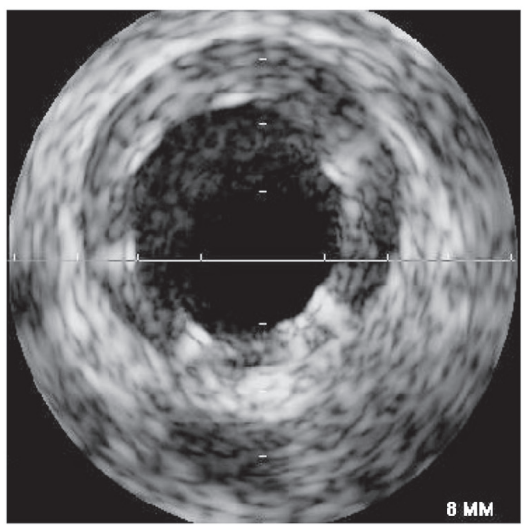


bolus injection of $60 \mathrm{~mL}$ of nonionic contrast media (Ultravist $370^{\circledR}$, Schering) followed by a $60 \mathrm{~mL}$ saline bolus injection, and both were injected at a flow rate of $4 \mathrm{~mL} / \mathrm{second}$. All CT images were analyzed by a radiologist who had 5 years experience with coronary CTA imaging. The axial images were reconstructed at multiple phases that covered the cardiac cycle in increments of $10 \%$ of the RR interval between 5\% and 95\%. Multiphase reconstruction was performed using short axis slices from the base to the apex of the heart using commercially available software (Argus ${ }^{\circledR}$, Siemens) (Figure 1).

Conventional coronary angiography: CCA was performed using a digital flat panel fluoroscope (Phillips) via a femoral approach applying nonionic contrast material (Ultravist ${ }^{\circledR} 370$, Schering, Berlin, Germany). A minimum of 4 orthogonal views were obtained (Figure 2).

Statistical analysis: Continuous variables with normal distributions are expressed as the mean \pm SD and were compared using an unpaired Student's $t$-test. Categorical variables were compared using the chi-square test, where appropriate. Relative risks were calculated by dividing the Kaplan-Meier estimated rate of factors in the false negative group by that in the true negative group. The 95 percent confidence interval for the relative risk was calculated using the standard errors from the Kaplan-Meier curve. The predictive factors for significant stenosis were calculated by multiple logistic regression analysis. A $P$ value less than 0.05 was deemed as being significant. Statistical analyses were performed with Statistical Package for Social Sciences software (SPSS 15.0 for Windows).

\section{RESULTS}

Clinical characteristics: The mean age was higher in group I than in group II (63 \pm 6 versus $52 \pm 12$ years, $P<0.001$ ). Sex and body mass index were not significantly different between the groups. There were higher incidences of a history of hypertension $(64.3 \%$ versus $7.9 \%, P<0.001)$ and current smoking $(35.7 \%$ versus $3.9 \%, P<0.001)$ in group I. There were no significant differences in the incidences of diabetes and hyperlipidemia between the groups. Past pharmacological therapy was similar between the groups; $28.6 \%$ of group I and $17.1 \%$ of group II had taken aspirin, $14.3 \%$ and $7.9 \%$ clopidogrel, $21.4 \%$ and $18.4 \%$ betablockers, $14.3 \%$ and $7.9 \%$ angiotensin converting enzyme inhibitors, $14.3 \%$ and $11.8 \%$ angiotensin receptor blockers, and $14.3 \%$ and $14.5 \%$ statins (Table I).

Laboratory findings: The lipid profiles were not significantly different between the groups (Table II). The levels of uric acid and homocysteine were higher in group I than in group II ( $P=0.008, P=0.010$, respectively). The levels of fibrinogen and high sensitivity C-reactive protein (hs-CRP) were not significantly different between the groups. The levels of NT-proBNP and cardiac enzymes 
Table I. Baseline Clinical Characteristics

\begin{tabular}{lccr}
\hline & Group I $(n=14)$ & Group II $(n=76)$ & $P$ \\
\hline Age (years) & $62.6 \pm 7.5$ & $52.1 \pm 12.0$ & $<0.001$ \\
Male (\%) & $7(50.0)$ & $42(55.3)$ & 0.735 \\
Body mass index $\left(\mathrm{kg} / \mathrm{m}^{2}\right)$ & $24.9 \pm 12.7$ & $24.6 \pm 7.5$ & 0.744 \\
Risk factor (\%) & & & \\
$\quad$ Hypertension & $9(64.3)$ & $6(7.9)$ & $<0.001$ \\
Diabetes mellitus & $0(0.0)$ & $2(2.6)$ & 0.539 \\
Smoking & $5(35.7)$ & $3(3.9)$ & $<0.001$ \\
Hyperlipidemia & $2(14.3)$ & $2(2.6)$ & 0.052 \\
Family history & $2(14.3)$ & $6(7.9)$ & 0.298 \\
Past medication $(\%)$ & & & \\
Aspirin & $4(28.6)$ & $13(17.1)$ & 0.314 \\
Clopidogrel & $2(14.3)$ & $6(7.9)$ & 0.440 \\
Beta-blocker & $3(21.4)$ & $14(18.4)$ & 0.792 \\
Angiotensin converting enzyme inhibitor & $2(14.3)$ & $6(7.9)$ & 0.440 \\
Angiotensin receptor blocker & $2(14.3)$ & $9(11.8)$ & 0.798 \\
Statin & $2(14.3)$ & $11(14.5)$ & 0.985 \\
\hline
\end{tabular}

Table II. Laboratory Findings

\begin{tabular}{lccc}
\hline & Group I $(n=14)$ & Group II $(n=76)$ & $P$ \\
\hline Lipid profiles & & & \\
Total cholesterol & $182.5 \pm 50.3$ & $172.1 \pm 41.3$ & 0.589 \\
Low density lipoprotein & $130.6 \pm 43.0$ & $111.1 \pm 36.8$ & 0.287 \\
High density lipoprotein & $61.8 \pm 25.3$ & $50.4 \pm 15.1$ & 0.080 \\
Triglycerides & $130.3 \pm 41.1$ & $106.8 \pm 83.0$ & 0.203 \\
Apolipoprotein A & $153.7 \pm 39.6$ & $140.6 \pm 26.0$ & 0.121 \\
Apolipoprotein B1 & $98.1 \pm 31.8$ & $103.0 \pm 93.5$ & 0.732 \\
Lipoprotein(a) & $24.4 \pm 16.0$ & $31.4 \pm 25.1$ & 0.192 \\
Inflammatory markers & & & \\
Uric acid & $5.7 \pm 1.5$ & $4.6 \pm 1.2$ & 0.008 \\
Homocysteine & $9.6 \pm 3.1$ & $7.4 \pm 2.5$ & 0.010 \\
Fibrinogen & $256.7 \pm 48.6$ & $271.4 \pm 67.3$ & 0.568 \\
Highly sensitive C-reactive protein & $0.4 \pm 1.2$ & $0.3 \pm 0.2$ & 0.708 \\
Cardiac enzymes & & & \\
Creatine kinase & $93.1 \pm 46.7$ & $89.5 \pm 40.2$ & 0.824 \\
Creatine kinase-MB & $5.2 \pm 3.4$ & $5.6 \pm 3.1$ & 0.729 \\
Troponin I & $0.02 \pm 0.02$ & $0.04 \pm 0.08$ & 0.306 \\
Troponin T & $0.01 \pm 0.01$ & $0.01 \pm 0.0$ & 0.370 \\
\hline
\end{tabular}

[creatine kinase (CK), CK-MB, troponin $\mathrm{T}$ and I] were not significantly different between the groups.

Electrocardiographic and echocardiographic findings: ST segment depression or T-wave inversion was observed on the ECG in 5 patients in group I and in 1 patient in group II $(35.7 \%$ versus $1.3 \%, P<0.001)$. The ejection fraction was $69.1 \%$ in group I and $66.6 \%$ in group II (Table III).

CCA findings in group I: In 14 patients, the vessel involved was the left anterior 
Table III. Electrocardiogram and Echocardiogram Findings

\begin{tabular}{lccc}
\hline & Group I $(n=14)$ & Group II $(n=76)$ & $P$ \\
\hline $\begin{array}{l}\text { Electrocardiogram findings } \\
\quad \text { Within normal limits }\end{array}$ & $9(64.3)$ & $75(98.7)$ & $<0.001$ \\
$\quad \begin{array}{l}\text { ST segment depression or T inversion } \\
\text { Echocardiogram findings }\end{array}$ & $5(35.7)$ & $1(1.3)$ & \\
$\quad$ Ejection fraction & $69.1 \pm 7.5$ & $66.6 \pm 5.7$ & 0.174 \\
\hline
\end{tabular}

Table IV. Conventional Coronary Angiographic Findings

\begin{tabular}{lc}
\hline & Group I $(n=14)$ \\
\hline Artery of stenosis $(\%)$ & $0(0.0)$ \\
Left main & $9(64.3)$ \\
Left anterior descending artery & $1(7.1)$ \\
Left circumflex artery & $4(28.6)$ \\
Right coronary artery & \\
Lesion type* $\%)$ & $0(0.0)$ \\
A & $4(28.6)$ \\
B1 & $7(50.0)$ \\
B2 & $3(21.4)$ \\
C & $86.7 \pm 14.2$ \\
Diameter stenosis $(\%)$ & \\
TIMI flow (\%) & $1(7.1)$ \\
0 or 1 & $5(35.6)$ \\
2 & $8(57.3)$ \\
3 & \\
Percutaneous coronary intervention & $14(100.0)$ \\
Success rate $(\%)$ & $1(7.1)$ \\
Bare metal stent $(\%)$ & $5(35.6)$ \\
Sirolimus-eluting stent $(\%)$ & $9(64.3)$ \\
Paclitaxel-eluting stent $(\%)$ & $3.6 \pm 1.4$ \\
Stent diameter $($ mm) & $25.7 \pm 7.9$ \\
Stent length (mm) & \\
\hline
\end{tabular}

*Lesion type according to ACC/AHA

descending artery in 9 patients, the right coronary artery in 4 patients, and the left circumflex artery in 1 patient. Lesion type according to the ACC/AHA criteria was type B1 in 4 patients, B2 in 7, and type $\mathrm{C}$ in 3. Mean diameter stenosis was $86.7 \pm 14.2 \%$. Pre-PCI thrombolysis in myocardial infarction (TIMI) flow was 1 in one patient, 2 in 5 patients, and 3 in 3 patients.

All patients underwent PCI with stent implantation. A bare metal stent was used in 1 patient, sirolimus-eluting stents in 5 patients, and paclitaxel-eluting stents in 9 patients. Mean stent diameter was $3.6 \pm 1.4 \mathrm{~mm}$ and mean stent length was $25.7 \pm 7.8 \mathrm{~mm}$ (Table IV).

Predictors for significant stenosis in CCA with normal MDCT: A past history of hypertension, high uric acid levels, and ST segment depression or T wave inver- 
Table V. Multivariate Analysis of Significant Coronary Artery Stenosis in Conventional Coronary Angiogram

\begin{tabular}{lrrrr} 
& & \multicolumn{2}{c}{$95 \%$ confidence interval } & \\
\cline { 3 - 4 } & Odd ratio & $P$ \\
\cline { 3 - 4 } & & Lower & Upper & \\
\hline Hypertension & 11.11 & 4.67 & 10.00 & 0.009 \\
High level of uric acid & 4.76 & 1.41 & 1.61 & 0.012 \\
ST segment depression or T inversion & 1.81 & 1.05 & 3.33 & 0.046 \\
$\quad$ in electrocardiogram & 1.10 & 0.09 & 6.22 & 0.097 \\
Old age & 1.26 & 0.92 & 1.73 & 0.161 \\
High level of homocysteine & 1.49 & 0.69 & 3.25 & 0.314 \\
High body mass index & 0.42 & 0.16 & 1.09 & 0.601 \\
Statin treatment & 1.03 & 0.68 & 2.05 & 0.678 \\
High level of hs-CRP & 0.97 & 0.79 & 1.17 & 0.738 \\
Ejection fraction & 1.10 & 0.77 & 1.85 & 0.789 \\
High level of NT-pro BNP & 1.10 & 0.88 & 1.74 & 0.881 \\
Diabetes & & & \\
\hline
\end{tabular}

hs-CRP indicates high sensitivity C-reactive protein; and NT-pro BNP, N-terminal pro brain natriuretic peptide.

sion in ECG are independent predictive factors for significant stenosis in CCA ( $P=0.009, P=0.012, P=0.046$, respectively) in patients having normal MDCT findings (Table V).

\section{Discussion}

To the best of our knowledge, this is the first study to show that the findings of coronary stenosis by MDCT do not always correlate with that of CCA in certain situations.

It is known that anatomical coronary artery disease, such as that assessed by CCA, does not always correlate with the functional severity of the disease. ${ }^{9-12)}$

A recent study has demonstrated how much the latest improvement in MDCT technology with a faster gantry rotation time and submillimeter slices acquired with 64-slice CT scanners has led to an increase in segments of the coronary tree being evaluable for the detection of coronary artery stenosis. MDCT has been shown to be a reliable diagnostic technique for the evaluation of coronary artery stenoses. ${ }^{10-13)}$ However, coronary calcification influences image quality, and blurring due to severe calcification can cause an image to be uninterpretable. ${ }^{14-18)}$ Furthermore, MDCT tends to overestimate the degree of stenosis compared with that of CCA..$^{10,11)}$

Until now, Medline searches have not identified any reports that analyzed the characteristics of patients with false negatives using MDCT.

This study has several limitations. First, the study population was very small. Only 14 patients were enrolled in the false negative group. As mentioned 
above, because of the high negative predictive value of MDCT, whose maximum is $99 \%$, the frequency of discordance between MDCT and CCA is very low. Multicenter trials are necessary to overcome this limitation. Second, assessment of diameter stenosis was only performed by QCA. Intravascular ultrasonography (IVUS) has advantages for the evaluation of coronary atherosclerotic burden and disease progression. ${ }^{19-23)}$ Third, our study was a retrospective and crosssectional study, and not a randomized and controlled prospective study. Finally, all patients with normal MDCT findings did not undergo CCA. The sensitivity, specificity, positive predictive value, and negative predictive value could not be calculated in our study.

CCA may be necessary in patients who complain of persistent chest pain with hypertension, high levels of uric acid, and ischemic evidence in electrocardiograms, although having normal findings in MDCT. Although MDCT appears to be useful as a screening tool for patients with suspected coronary artery disease, it does not yet seem to have a complementary role in clinical decisionmaking for revascularization strategies.

Prospective studies involving many patients are warranted to define the characteristics of patients with a false negative in MDCT in clinical practice.

\section{REFERENCES}

1. Mosca L, Grundy SM, Judelson D, et al. Guide to Preventive Cardiology for women. AHA/ACC Scientific Statement Consensus panel statement. Circulation 1999; 99: 2480-4. (Review)

2. Wake R, Takeuchi M, Yoshikawa J, Yoshiyama M. Effects of gender on prognosis of patients with known or suspected coronary artery disease undergoing contrast-enhanced dobutamine stress echocardiography. Circ J 2007; 71: 1060-6.

3. Bashore TM, Bates ER, Berger PB, et al; American College of Cardiology. Task Force on Clinical Expert Consensus Documents. American College of Cardiology/Society for Cardiac Angiography and Interventions Clinical Expert Consensus Document on cardiac catheterization laboratory standards. A report of the American College of Cardiology Task Force on Clinical Expert Consensus Documents. J Am Coll Cardiol 2001; 37: 2170-214. (Review)

4. Garcia MJ, Lessick J, Hoffmann MH; CATSCAN Study Investigators. Accuracy of 16-row multidetector computed tomography for the assessment of coronary artery stenosis. JAMA 2006; 26: 403-11.

5. Hara T, Hayashi T, Izawa I, Kajiya T. Noninvasive detection of Takotsubo [corrected] cardiomyopathy using multi-detector row computed tomography. Int Heart J 2007; 48: 773-8.

6. Rubinshtein R, Halon DA, Gaspar T, et al. Usefulness of 64-slice cardiac computed tomographic angiography for diagnosing acute coronary syndromes and predicting clinical outcome in emergency department patients with chest pain of uncertain origin. Circulation 2007; 115: 1762-8.

7. Reant $\mathrm{P}$, Brunot $\mathrm{S}$, Lafitte $\mathrm{S}$, et al. Predictive value of noninvasive coronary angiography with multidetector computed tomography to detect significant coronary stenosis before valve surgery. Am J Cardiol 2006; 97: 1506-10.

8. Gaudio C, Mirabelli F, Peliccia F, et al. Early detection of coronary artery disease by 64-slice multidetector computed tomography in asymptomatic hypertensive high-risk patients. Int J Cardiol (in press).

9. Mühlenbruch G, Seyfarth T, Soo CS, Pregalathan N, Mahnken AH. Diagnostic value of 64-slice multidetector row cardiac CTA in symptomatic patients. Eur Radiol 2007; 17: 603-9.

10. Halon DA, Rubinshtein R, Gaspar T, Peled N, Lewis BS. Current status and clinical applications of 
cardiac multidetector computed tomography. Cardiology 2008; 109: 73-84. (Review)

11. Groen JM, Greuter MJ, van Ooijen PM, Oudkerk M. A new approach to the assessment of lumen visibility of coronary artery stent at various heart rates using 64-slice MDCT. Eur Radiol 2007; 17: 1879-84.

12. Butler J, Shapiro MD, Jassal DS, et al. Comparison of multidetector computed tomography and twodimensional transthoracic echocardiography for left ventricular assessment in patients with heart failure. Am J Cardiol 2007; 99:247-9.

13. Onuma Y, Tanabe K, Nakazawa G, et al. Noncardiac findings in cardiac imaging with multidetector computed tomography. J Am Coll Cardiol 2006; 48: 402-6.

14. Dorgelo J, Willems TP, van Ooijen PM, et al. A 16-slice multidetector computed tomography protocol for evaluation of the gastroepiploic artery grafts in patients after coronary artery bypass surgery. Eur Radiol 2005; 15: 1994-9.

15. Kefer J, Coche E, Legros G, et al. Head-to-head comparison of three-dimensional navigator-gated magnetic resonance imaging and 16-slice computed tomography to detect coronary artery stenosis in patients. J Am Coll Cardiol 2005; 46: 92-100.

16. Becker CR, Knez A, Leber A, et al. Detection of coronary artery stenoses with multislice helical CT angiography. J Comput Assist Tomogr 2002; 26: 750-5.

17. Kuettner A, Kopp AF, Schroeder S, et al. Diagnostic accuracy of multidetector computed tomography coronary angiography in patients with angiographically proven coronary artery disease. J Am Coll Cardiol 2004; 43: 831-9.

18. Butler J, Shapiro M, Reiber J, et al. Extent and distribution of coronary artery disease: a comparative study of invasive versus noninvasive angiography with computed angiography. Am Heart J 2007; 153: 378-84.

19. Böse D, von Birgelen C, Erbel R. Intravascular ultrasound for the evaluation of therapies targeting coronary atherosclerosis. J Am Coll Cardiol 2007; 49: 925-32. (Review)

20. Orford JL, Lerman A, Holmes DR. Routine intravascular ultrasound guidance of percutaneous coronary intervention: a critical reappraisal. J Am Coll Cardiol 2004; 43: 1335-42. (Review)

21. Uemura R, Tanabe J, Yokoyama H, Ohaki M. Impact of histological plaque characteristics on intravascular ultrasound parameters at culprit lesions in coronary artery disease. Int Heart J 2006; 47: 683-93.

22. Schrale RG, Channon KM, Ormerod OJ. IVUS-guided evaluation and percutaneous intervention in an anomalous left main coronary artery. J Invasive Cardiol 2007; 19: E195-8.

23. Brown BG, Zhao XQ. Is intravascular ultrasound the gold standard surrogate for clinically relevant atherosclerosis progression? J Am Coll Cardiol 2007; 49: 933-8. (Review) 